\title{
On some fixed-point theorems for $\psi$-contraction on metric space involving a graph
}

\author{
Mahpeyker Öztürk* and Ekber Girgin
}

\section{"Correspondence:}

mahpeykero@sakarya.edu.tr Department of Mathematics, Sakarya University, Sakarya, 54187, Turkey

\begin{abstract}
In this paper, we introduce the $(G, \psi)$-contraction and the $(G, \psi)$-graphic contraction in a metric space by using a graph. We explain some conditions for a mapping which is a $(G, \psi)$-contraction to have a unique fixed point and also we give conditions as regards the existence of a fixed point for $(G, \psi)$-graphic contraction by applying the connectivity of the graph in both cases. Moreover, we give examples to show that our results are a substantial improvement of some known results in the literature.
\end{abstract}

MSC: $47 \mathrm{H} 10 ; 54 \mathrm{H} 25$

Keywords: connected graph; fixed point; metric space; $\psi$-type contraction

\section{Introduction}

The metric fixed-point theory has been researched extensively in the past two decades such as in a metric space endowed with a partial ordering, and many results appeared giving sufficient conditions for a mapping to be a Picard operator. For these concepts have been given two main theorems, which are the Banach Contraction Principle and the Knaster-Tarski Theorem [1].

Recently Jachymski [2] and Gwóźdź-Lukawska and Jachymski [3] have given an interesting concept in fixed-point theory with some general structures by using the context of metric spaces endowed with a graph. Jachymski [2] has proved some generalizations of the Banach Contraction Principle to mappings on a metric space endowed with a graph and also has presented its applications to the Kelisky-Rivlin Theorem on iterates of the Bernstein operators on the space $C[0,1]$. Afterwards different contractions have been studied by various authors. In [4] the contraction principle for set-valued mappings, in [5-7] Kannan type, Reich type contractions, and $\varphi$-contractions have been investigated, respectively. Some new fixed-point results for graphic contractions on a complete metric space with a graph have been presented in [8]; also they gave a particular case of almost contractions.

In this paper, motivated by the work of Jachymski [2] and Petruşel [8], we introduce new contractions for the mappings on complete metric space and prove some fixed-point theorems. Our results generalize and unify some results by the above-mentioned authors.

\section{Basic facts and definitions}

Let $(X, d)$ be a metric space and $\Delta$ denote the diagonal of the Cartesian product $X \times X$. Let $G$ be a directed graph such that the set $V(G)$ of its vertices coincides with $X$, and the

\section{黛 Springer}

○2014 Öztürk and Girgin; licensee Springer. This is an Open Access article distributed under the terms of the Creative Commons Attribution License (http://creativecommons.org/licenses/by/2.0), which permits unrestricted use, distribution, and reproduction in any medium, provided the original work is properly cited. 
set $E(G)$ of its edges contains all loops; that is, $E(G) \supseteq \Delta$. Assume that $G$ has no parallel edges, so one can identify $G$ with the pair $(V(G), E(G)$ ).

The conversion of a graph $G$ is denoted by $G^{-1}$ and this is a graph obtained from $G$ by reversing the direction of the edges. Hence

$$
E\left(G^{-1}\right)=\{(x, y) \in X \times X:(y, x) \in E(G)\} .
$$

By $\tilde{G}$ we denote the undirected graph obtained from $G$ by omitting the direction of the edges. Indeed, it is more convenient to treat $\tilde{G}$ as a directed graph for which the set of its edges is symmetric, and under this convention, we have

$$
E(\tilde{G})=E(G) \cup E\left(G^{-1}\right) .
$$

A subgraph of a graph $G$ is a graph $H$ such that $V(H) \subseteq V(G)$ and $E(H) \subseteq E(G)$. Let $x$ and $y$ be vertices in a graph $G$. A path from $x$ to $y$ of length $N(N \in \mathbf{N} \cup\{0\})$ is a sequence $\left(x_{i}\right)_{i=0}^{N}$ of $N+1$ distinct vertices such that $x_{0}=x, x_{N}=y$ and $\left(x_{i-1}, x_{i}\right) \in E(G)$ for $i=1, \ldots, N$. The number edges in $G$ forming the path is called the length of the path. A graph $G$ is connected if there is a path between any two vertices. If a graph $G$ is not connected then it is called disconnected and its different paths are called the components of $G$. Every component of $G$ is a subgraph of it. Furthermore, $G$ is weakly connected if $\tilde{G}$ is connected. Let $G_{x}$ be the component of $G$ which consists of all edges and vertices contained in some path in $G$ beginning at $x$. Suppose that $G$ is such that $E(G)$ is symmetric; then $V(G)=[x]_{G}$ where $[x]_{G}$ denotes the equivalence class of relations $\Re$ defined on $V(G)$ by the rule

$y \Re z$ if there is a path in $G$ from $y$ to $z$.

Some basic notations related to connectivity of graphs can be found in [9].

If $f: X \rightarrow X$ is an operator, then we denote by

$$
F(f)=\{x \in X: x=f x\}
$$

the set of all fixed points of $f$.

Definition 1 [2] A mapping $f: X \rightarrow X$ is a Banach $G$-contraction or simply $G$-contraction if $f$ preserves edges of $G$;

$$
(x, y) \in E(G) \quad \Rightarrow \quad(f x, f y) \in E(G),
$$

for all $x, y \in X$, and $f$ decreases weights of edges of $G$ : for all $x, y \in X$ there exists $\alpha \in(0,1)$ such that

$$
(x, y) \in E(G) \quad \Rightarrow \quad d(f x, f y)<\leq \alpha d(x, y) .
$$

Definition 2 [8] The mapping $f: X \rightarrow X$ is a G-graphic contraction

(i) if $f$ preserves edges of $G$;

$$
(x, y) \in E(G) \quad \Rightarrow \quad(f x, f y) \in E(G),
$$

for all $x, y \in X$; 
(ii) there exists $\alpha \in(0,1)$ such that

$$
(x, y) \in E(G) \quad \Rightarrow \quad d\left(f x, f^{2} x\right) \leq \alpha d(x, f x)
$$

for all $x, y \in X_{f}$.

Definition 3 [2] A mapping $f: X \rightarrow X$ is called orbitally continuous if for all $x, y \in X$ and any sequence $\left(k_{n}\right)_{n \in \mathbf{N}}$ of positive integers,

$$
f^{k_{n}} x \rightarrow y \quad \text { implies } \quad f\left(f^{k_{n}} x\right) \rightarrow f y \quad \text { as } n \rightarrow \infty
$$

Definition 4 [2] A mapping $f: X \rightarrow X$ is called orbitally G-continuous if for all $x, y \in X$ and any sequence $\left(k_{n}\right)_{n \in \mathbf{N}}$ of positive integers,

$$
f^{k_{n}} x \rightarrow y, \quad\left(f^{k_{n}} x, f^{k_{n+1}} x\right) \in E(G) \quad \text { imply } \quad f\left(f^{k_{n}} x\right) \rightarrow f y \quad \text { as } n \rightarrow \infty
$$

Now, we give a definition of the class $\Psi$ which is used in several well-known papers to obtain some fixed-point results [10-13].

Definition 5 Let us define the class $\Psi=\left\{\psi: \mathbf{R}^{+} \rightarrow \mathbf{R}^{+} \mid \psi\right.$ is nondecreasing $\}$ which satisfies the following conditions:

(i) $\psi(\omega)=0$ if and only if $\omega=0$;

(ii) for every $\left(\omega_{n}\right) \in \mathbf{R}^{+}, \psi\left(\omega_{n}\right) \rightarrow 0$ if and only if $\omega_{n} \rightarrow 0$;

(iii) for every $\omega_{1}, \omega_{2} \in \mathbf{R}^{+}, \psi\left(\omega_{1}+\omega_{2}\right) \leq \psi\left(\omega_{1}\right)+\psi\left(\omega_{2}\right)$.

\section{$3(G, \psi)$-Contraction and related fixed-point theorems}

We establish some fixed-point theorems in metric space with a graph by defining the $(G, \psi)$-contraction.

Definition 6 We say that a mapping $f: X \rightarrow X$ is a $(G, \psi)$-contraction if the following hold;

(i) $f$ preserves edges of $G$, i.e. $((x, y) \in E(G) \Rightarrow(f x, f y) \in E(G)), \forall x, y \in X$;

(ii) $f$ decreases the weight of edges of $G$, that is, there exists $c \in(0,1)$ such that

$$
(x, y) \in E(G) \quad \Rightarrow \quad \psi(d(f x, f y)) \leq c \psi(d(x, y))
$$

for all $x, y \in X$.

Lemma 1 Iff $: X \rightarrow X$ is a $(G, \psi)$-contraction, then $f$ is both $a\left(G^{-1}, \psi\right)$-contraction and a $(\tilde{G}, \psi)$-contraction.

Proof The proof can be obtained by the symmetry of $d$ and the definition of the $(\tilde{G}, \psi)$ contraction.

Lemma 2 Let $f: X \rightarrow X$ be a $(G, \psi)$-contraction with constant $c \in(0,1)$; for a given $x \in X$ and $y \in[x]_{\tilde{G}}$, there exists $r(x, y) \geq 0$ such that

$$
\psi\left(d\left(f^{n} x, f^{n} y\right)\right) \leq c^{n} r(x, y)
$$


Proof Let $x \in X$ and $y \in[x]_{\tilde{G}}$. Then there is a path $\left(x_{i}\right)_{i=0}^{N}$ in $\tilde{G}$ from $x$ to $y$, which means $x_{0}=x, x_{N}=y$, and $\left(x_{i-1}, x_{i}\right) \in E(\tilde{G})$ for $i=1,2, \ldots, N$. By Lemma $1, f$ is a $(\tilde{G}, \psi)$-contraction. With an easy induction, we have $\left(f^{n} x_{i-1}, f^{n} x_{i}\right) \in E(\tilde{G})$ and

$$
\begin{aligned}
\psi\left(d\left(f^{n} x_{i-1}, f^{n} x_{i}\right)\right) & \leq c \psi\left(d\left(f^{n-1} x_{i-1}, f^{n-1} x_{i}\right)\right) \\
& \leq c\left(c \psi\left(d\left(f^{n-2} x_{i-1}, f^{n-2} x_{i}\right)\right)\right) \leq \cdots \leq c^{n} \psi\left(d\left(x_{i-1}, x_{i}\right)\right)
\end{aligned}
$$

for all $n \in \mathbf{N}$ and $i=1,2, \ldots, N$.

Hence using the triangle inequality, we get

$$
\psi\left(d\left(f^{n} x, f^{n} y\right)\right) \leq \sum_{i=1}^{N} \psi\left(d\left(f^{n} x_{i-1}, f^{n} x_{i}\right)\right) \leq c^{n} \sum_{i=1}^{N} \psi\left(d\left(x_{i-1}, x_{i}\right)\right)
$$

So it qualifies to set $r(x, y):=\sum_{i=1}^{N} \psi\left(d\left(x_{i-1}, x_{i}\right)\right)$.

Lemma 3 Let $(X, d)$ be a complete metric space endowed with a graph $G$ and $f: X \rightarrow X$ be $a(G, \psi)$-contraction for which there exists $x_{0} \in X$ such that $f x_{0} \in\left[x_{0}\right]_{\tilde{G}}$. Let $\tilde{G}_{x_{0}}$ be the component of $\tilde{G}$ containing $x_{0}$. Then $\left[x_{0}\right]_{\tilde{G}}$ is $f$-invariant and $\left.f\right|_{[x] \tilde{G}}$ is a $\left(\tilde{G}_{x_{0}}, \psi\right)$-contraction. Furthermore, $x, y \in\left[x_{0}\right]_{\tilde{G}}$, and the sequences $\left(f^{n} x\right)_{n \in \mathbf{N}}$ and $\left(f^{n} y\right)_{n \in \mathbf{N}}$ are Cauchy equivalent.

Proof The proof of this lemma can obtained by using similar arguments as given in [7]. So we omit the proof.

The following result shows that there is a close relation between convergence of an iteration sequence which can be obtained by using a $(G, \psi)$-contraction mapping and connectivity of the graph.

Theorem 1 Let $(X, d)$ be a metric space endowed with a graph $G$ and $f: X \rightarrow X$ be a $(G, \psi)$-contraction, then the following statements are equivalent:

(i) $G$ is weakly connected;

(ii) for given $x, y \in X$, the sequences $\left(f^{n} x\right)_{n \in \mathbf{N}}$ and $\left(f^{n} y\right)_{n \in \mathbf{N}}$ are Cauchy equivalent;

(iii) $\operatorname{card} F(f) \leq 1$.

Proof (i) $\Rightarrow$ (ii) Let $f$ be a $(G, \psi)$-contraction and $x, y \in X$. By hypothesis, $[x]_{\tilde{G}}=X$, so $f x \in[x]_{\tilde{G}}$. By Lemma 2, we get

$$
\psi\left(d\left(f^{n} x, f^{n+1} x\right)\right) \leq c^{n} r(x, f x)
$$

for all $n \in \mathbf{N}$. Hence

$$
\sum_{n=0}^{\infty} \psi\left(d\left(f^{n} x, f^{n+1} x\right)\right)<\infty
$$

and if we use a standard argument, then $\left(f^{n} x\right)_{n \in \mathbf{N}}$ is obtained as a Cauchy sequence. Since also $y \in[x]_{\tilde{G}}$, Lemma 2 leads to $\psi\left(d\left(f^{n} x, f^{n} y\right)\right) \leq c^{n} r(x, y)$. Therefore, $\left(f^{n} x\right)_{n \in \mathbf{N}}$ and $\left(f^{n} y\right)_{n \in \mathbf{N}}$ are equivalent. Clearly, because $\left(f^{n} x\right)_{n \in \mathbf{N}}$ is a Cauchy sequence, so is $\left(f^{n} y\right)_{n \in \mathbf{N}}$. 
(ii) $\Rightarrow$ (iii) Let $f$ be a $(G, \psi)$-contraction and $x, y \in F(f)$. By (ii), $\left(f^{n} x\right)_{n \in \mathbf{N}}$ and $\left(f^{n} y\right)_{n \in \mathbf{N}}$ are equivalent, which yields $x=y$.

(iii) $\Rightarrow$ (ii) Suppose, to the contrary, $G$ is not weakly connected, that is, $\tilde{G}$ is disconnected. Let $x_{0} \in X$. Then the sets $\left[x_{0}\right]_{\tilde{G}}$ and $X-\left[x_{0}\right]_{\tilde{G}}$ both are nonempty. Let $y_{0} \in X-\left[x_{0}\right]_{\tilde{G}}$ and define

$$
f x= \begin{cases}x_{0}, & \text { if } x \in\left[x_{0}\right]_{\tilde{G}} \\ y_{0}, & \text { if } x \in X-\left[x_{0}\right]_{\tilde{G}} .\end{cases}
$$

Obviously, $F(f)=\left\{x_{0}, y_{0}\right\}$. We show $f$ is a $(G, \psi)$-contraction. Let $(x, y) \in E(G)$. Then $[x]_{\tilde{G}}=$ $[y]_{\tilde{G}}$, so either $x, y \in\left[x_{0}\right]_{\tilde{G}}$ or $x, y \in X-\left[x_{0}\right]_{\tilde{G}}$. Hence in both cases $f x=f y$, so $(f x, f y) \in E(G)$ as $E(G) \supseteq \Delta$, and $\psi(d(f x, f y))=0$. Thereby, $f$ is a $(G, \psi)$-contraction having two fixed points which violates the assumption.

The following result is an easy consequence of Theorem 1 .

Corollary 1 Let $(X, d)$ be a complete metric space endowed with a graph $G$ and $f: X \rightarrow X$ be a $(G, \psi)$-contraction, then the following statements are equivalent:

(i) $G$ is weakly connected;

(ii) there is $x^{*} \in X$ such that $\lim _{n \rightarrow \infty} f^{n} x=x^{*}$, for all $x \in X$.

Now, we give an example of $f$ being a $(G, \psi)$-contraction and this example shows that we could not add that $x^{*}$ is a fixed point of $f$ in Corollary 1.

Example 1 Let $X=[0,1]$ be endowed with the usual metric. Take

$$
E(G)=\{(0,0)\} \cup\{(0,1)\} \cup\{(x, y) \in(0,1] \times(0,1]: x \geq y\}
$$

and $f: X \rightarrow X$ as follows:

$$
f x= \begin{cases}\frac{x}{3}, & \text { if } x \in(0,1], \\ \frac{1}{2}, & \text { if } x=0 .\end{cases}
$$

Then $f$ is a $(G, \psi)$-contraction where $\psi(\omega)=\frac{\omega}{\omega+1}$.

Proof It can be easily seen that $G$ is a weakly connected graph and $f$ is a $(G, \psi)$-contraction where $\psi(\omega)=\frac{\omega}{\omega+1}$. It is a fact that $\left(f^{n} x\right) \rightarrow 0$, for all $x \in X$ but $f$ has no fixed point.

For any mapping which satisfies the condition of Corollary 1 to have a fixed point we need to add condition (6), which is given in the following theorem.

Theorem 2 Let $(X, d)$ be a complete metric space and the triple $(X, d, G)$ have the following condition:

$$
\text { for any }\left(x_{n}\right)_{n \in \mathbf{N}} \text { in } X \text {, if } x_{n} \rightarrow x \text { and }\left(x_{n}, x_{n+1}\right) \in E(G) \text { for } n \in \mathbf{N} \text {, }
$$$$
\text { then there is a subsequence }\left(x_{k_{n}}\right)_{n \in \mathbf{N}} \text { with }\left(x_{k_{n}}, x\right) \in E(G) \text { for } n \in \mathbf{N} \text {. }
$$

Let $f: X \rightarrow X$ be a $(G, \psi)$-contraction, and $X_{f}=\{x \in X:(x, f x) \in E(G)\}$. Then the following statements hold. 
(i) $\operatorname{card} F(f)=\operatorname{card}\left\{[x]_{\tilde{G}}: x \in X_{f}\right\}$.

(ii) $F(f) \neq \emptyset$ iff $X_{f} \neq \emptyset$.

(iii) $f$ has a unique fixed point iff there exists $x_{0} \in X_{f}$ such that $X_{f} \subseteq\left[x_{0}\right]_{\tilde{G}}$.

(iv) For any $x \in X_{f},\left.f\right|_{[x]} \tilde{G}$ is a Picard operator.

(v) If $X_{f} \neq \emptyset$ and $G$ is weakly connected, then $f$ is a Picard operator.

(vi) If $X^{\prime}:=\bigcup\left\{[x]_{\tilde{G}}: x \in X_{f}\right\}$, then $\left.f\right|_{X^{\prime}}$ is a weakly Picard operator.

(vii) Iff $\subseteq E(G)$, then $f$ is a weakly Picard operator.

Proof Initially, we prove the items (iv) and (v). Take $x \in X_{f}$ and then $f x \in[x]_{\tilde{G}}$, so by Lemma 3, if $y \in[x]_{\tilde{G}}$, then $\left(f^{n} x\right)_{n \in \mathbf{N}}$ and $\left(f^{n} y\right)_{n \in \mathbf{N}}$ are Cauchy equivalent. Since $X$ is complete, $\left(f^{n} x\right)_{n \in \mathbf{N}}$ converges to some $x^{*} \in X$. It is obvious that $\lim _{n \rightarrow \infty} f^{n} y=x^{*}$. Then by using induction we get

$$
\left(f^{n} x, f^{n+1} x\right) \in E(G)
$$

for all $n \in \mathbf{N}$, since $(x, f x) \in E(G)$. By (6), there is a subsequence $\left(f^{k_{n}} x\right)_{n \in \mathbf{N}}$ such that $\left(f^{k_{n}} x, x^{*}\right) \in E(G)$ for all $n \in \mathbf{N}$. If we use (7), we conclude that $\left(x, f x, f^{2} x, \ldots, f^{k_{1}}, x^{*}\right)$ is a path in $G$ and also in $\tilde{G}$ from $x$ to $x^{*}$, and this means that $x^{*} \in[x]_{\tilde{G}}$. Since $f$ is a $(G, \psi)$ contraction we have

$$
\psi\left(d\left(f^{k_{n+1}} x, f x^{*}\right)\right) \leq c \psi\left(d\left(f^{k_{n}} x, x^{*}\right)\right)
$$

for all $n \in \mathbf{N}$. By taking the limit as $n \rightarrow \infty$, we deduce $f x^{*}=x^{*}$. Thereby, $\left.f\right|_{[x] \tilde{G}}$ is a Picard operator. Also, we conclude that $f$ is a Picard operator, when $[x]_{\tilde{G}}=X$, since there is weakly connectedness of $G$.

(vi) is obvious from (iv). For proof of (vii), if $f \subseteq E(G)$ then $X_{f}=X$ and so $X^{\prime}=X$ holds. Thus $f$ is a weakly Picard operator because of (vi).

Let us define a mapping to prove (i): $\rho(x)=[x]_{\tilde{G}}$ for all $x \in F(f)$. It is sufficient to show that $\rho: F(f) \rightarrow C=\left\{[x]_{\tilde{G}}: x \in X_{f}\right\}$ is a bijection. Because $E(G) \supseteq \Delta$, we deduce $F(f) \subseteq X_{f}$ and then $\rho(F(f)) \subseteq C$. Beside, if $x \in X_{f}$, then by (iv), $\lim _{n \rightarrow \infty} f^{n} x \in[x]_{\tilde{G}} \cap F(f)$, which implies $\rho\left(\lim _{n \rightarrow \infty} f^{n} x\right)=[x]_{\tilde{G}}$ and so $\rho$ is a surjective mapping. We show that $f$ is injective. Take $x_{1}, x_{2} \in F(f)$ which are such that $\rho\left(x_{1}\right)=\rho\left(x_{2}\right) \Rightarrow\left[x_{1}\right]_{\tilde{G}}=\left[x_{2}\right]_{\tilde{G}}$, then $x_{2} \in\left[x_{1}\right]_{\tilde{G}}$ and so, by (i),

$$
\lim _{n \rightarrow \infty} f^{n} x_{2} \in\left[x_{1}\right]_{\tilde{G}} \cap F(f)=\left\{x_{1}\right\}
$$

which gives $x_{1}=x_{2}$. Thus, $f$ is injective and this is the desired result. Finally, one can see that (ii) and (iii) are easy consequences of (i).

Corollary 2 Let $(X, d)$ be complete metric space and $(X, d, G)$ obey condition (6). The following are equivalent:

(i) $G$ is weakly connected;

(ii) every $(G, \psi)$-contraction $f: X \rightarrow X$ such that $\left(x_{0}, f x_{0}\right) \in E(G)$, for some $x_{0} \in X$, is a Picard operator;

(iii) for any $(G, \psi)$-contraction, $\operatorname{card} F(f) \leq 1$. 
Proof (i) $\Rightarrow$ (ii): This can be obtained directly from Theorem 2(v).

(ii) $\Rightarrow$ (iii): Let $f: X \rightarrow X$ be a $(G, \psi)$-contraction. If $X_{f}$ is empty, so is $F(f)$, because $F(f)$ is a subset of $X_{f}$. If $X_{f}$ is nonempty, then by (ii), $F(f)$ is singleton. In these two cases, $\operatorname{card} F(f) \leq 1$.

(iii) $\Rightarrow$ (i): This implication follows from Theorem 1 .

Remark 1 In the above results by taking $\psi(\omega)=\omega$, we obtain Corollary 3.2, which is given in [2].

\section{$4(G, \psi)$-Graphic contraction and fixed-point theorems}

Now, we define $(G, \psi)$-graphic contraction and give some results and examples.

Definition 7 Let $(X, d)$ be a metric space and $G$ be a graph. The mapping $f: X \rightarrow X$ is called a $(G, \psi)$-graphic contraction if the following conditions hold:

(i) $(x, y) \in E(G)$ implies $(f x, f y) \in E(G)$ ( $f$ is edge preserving);

(ii) there exists a $\psi \in \Psi$ with constants $c \in[0,1)$ such that

$$
\psi\left(d\left(f x, f^{2} x\right)\right) \leq c \psi(d(x, f x))
$$

for all $x \in X^{f}$, where $X^{f}:=\{x \in X:(x, f x) \in E(G)$ or $(f x, x) \in E(G)\}$.

Firstly, we give the following lemmas which can be proved as in the above section.

Lemma 4 If $: X \rightarrow X$ is a $(G, \psi)$-graphic contraction, then $f$ is both a $\left(G^{-1}, \psi\right)$-graphic contraction and $a(\tilde{G}, \psi)$-graphic contraction.

Lemma 5 Let $f: X \rightarrow X$ be a $(G, \psi)$-graphic contraction with constant $c \in[0,1)$. Then, given $x \in X^{f}$, there exists $r(x) \geq 0$ such that

$$
\psi\left(d\left(f^{n} x, f^{n+1} x\right)\right) \leq c^{n} r(x)
$$

for all $n \in \mathbf{N}$, where $r(x):=\psi(d(x, f x))$.

Lemma 6 Suppose that $f: X \rightarrow X$ is a $(G, \psi)$-graphic contraction. Then for each $x \in X^{f}$, there exists $x^{*} \in X$ such that the sequence $\left(f^{n} x\right)_{n \in \mathbf{N}}$ converges to $x^{*}$ as $n \rightarrow \infty$.

Proof Take an arbitrary element $x$ in $X^{f}$. By Lemma 5, we obtain

$$
\psi\left(d\left(f^{n} x, f^{n+1} x\right)\right) \leq c^{n} r(x)
$$

for all $n \in \mathbf{N}$. Therefore, $\sum_{n=0}^{\infty} \psi\left(d\left(f^{n} x, f^{n+1} x\right)\right)<\infty$ and so $\psi\left(d\left(f^{n} x, f^{n+1} x\right)\right) \rightarrow 0$; consequently using the property of $\psi$ we have $d\left(f^{n} x, f^{n+1} x\right) \rightarrow 0$. Then we say that $\left(f^{n} x\right)_{n \in \mathbf{N}}$ is a Cauchy sequence. By the completeness of $X$, there exists $x^{*} \in X$ such that $\left(f^{n} x\right)_{n \in \mathbf{N}}$ converges as $n \rightarrow \infty$.

Lemma 7 The self-mapping $f$ is a $(G, \psi)$-graphic contraction for which there exists $x_{0} \in X$ such that $f x_{0} \in\left[x_{0}\right]_{\tilde{G}}$. Then the set $\left[x_{0}\right]_{\tilde{G}}$ invariant with respect tof and $\left.f\right|_{\left[x_{0}\right]_{\tilde{G}}}$ is a $\left(\tilde{G}_{x_{0}}, \psi\right)$ graphic contraction, where $\tilde{G}_{x_{0}}$ is the component of $\tilde{G}$ containing $x_{0}$. 
Proof Let $x$ be an element in $\left[x_{0}\right]_{\tilde{G}}$. Then there exist $\left(x_{i}\right)_{i=0}^{N}$ in $\tilde{G}$ from $x_{0}$ to $x$, i.e., $x_{N}=x$ and $\left(x_{i-1}, x_{i}\right) \in E(\tilde{G})$ for $i=1,2, \ldots, N$. Since $f$ is a $(G, \psi)$-graphic contraction we get $\left(f x_{i-1}, f x_{i}\right) \in$ $E(\tilde{G})$ for $i=1,2, \ldots, N$. So we have a path from $f x_{0}$ to $f x$. Therefore $f x \in\left[f x_{0}\right]_{\tilde{G}}=\left[x_{0}\right]_{\tilde{G}}$ since $f x_{0} \in\left[x_{0}\right]_{\tilde{G}}$. Consequently $\left[x_{0}\right]_{\tilde{G}}$ is invariant with respect to $f$.

Take $(x, y) \in E\left(\tilde{G}_{x_{0}}\right)$; then there is a path $\left(x_{i}\right)_{i=0}^{N}$ in $\tilde{G}$ from $x_{0}$ to $y$ such that $x_{N-1}=x$. Also let $\left(y_{i}\right)_{i=0}^{M}$ be a path in $\tilde{G}$ from $x_{0}$ to $f x_{0}$. Then we realize

$$
\left(y_{0}, y_{1}, \ldots, y_{M}, f x_{1}, f x_{2}, \ldots, f x_{N-1}=f x, f x_{N}=f y\right)
$$

is a path in $\tilde{G}$ from $x_{0}$ to $f y$ such that $(f x, f y) \in E\left(\tilde{G}_{x_{0}}\right)$. Furthermore, $f$ is a $\left(\tilde{G}_{x_{0}}, \psi\right)$-graphic contraction because $E\left(\tilde{G}_{x_{0}}\right) \subseteq E(\tilde{G})$ and $f$ is a $(\tilde{G}, \psi)$-graphic contraction.

Theorem 3 Let $(X, d)$ be a complete metric space and let the triple $(X, d, G)$ have the following condition:

for any $\left(x_{n}\right)_{n \in \mathbf{N}}$ in $X$, if $x_{n} \rightarrow x$ and $\left(x_{n}, x_{n+1}\right) \in E(G)$

(or, respectively, $\left.\left(x_{n+1}, x_{n}\right) \in E(G)\right)$

for all $n \in \mathbf{N}$, then there is a subsequence $\left(x_{k_{n}}\right)_{n \in \mathbf{N}}$ with $\left(x_{k_{n}}, x\right) \in E(G)$

(or, respectively, $\left.\left(x, x_{k_{n}}\right) \in E(G)\right)$ for all $n \in \mathbf{N}$.

Let $f: X \rightarrow X$ be a $(G, \psi)$-graphic contraction and $f$ is orbitally G-continuous. Then the following statements hold:

(i) $F(f) \neq \emptyset$ if and only if $X^{f} \neq \emptyset$.

(ii) If $X^{f} \neq \emptyset$ and $G$ is weakly connected, then $f$ is a weakly Picard operator.

(iii) For any $x \in X^{f}$, we see that $\left.f\right|_{[x] \tilde{G}}$ is a weakly Picard operator.

Proof We begin with the statement (iii). Let $x \in X^{f}$; by Lemma 6 , there exists $x^{*} \in X$ such that $\lim _{n \rightarrow \infty} f^{n} x=x^{*}$. Since $x \in X^{f}$, then $f^{n} x \in X^{f}$ for every $n \in \mathbf{N}$. Now assume that $(x, f x) \in E(G)$. (A similar deduction can be made if $(f x, x) \in E(G)$.) By condition (9), there is a subsequence $\left(f^{k_{n}} x\right)_{n \in \mathbf{N}}$ of $\left(f^{n} x\right)_{n \in \mathbf{N}}$ such that $\left(f^{k_{n}} x, x^{*}\right) \in E(G)$ for each $n \in \mathbf{N}$. A path in $G$ can be formed by using the points $x, f x, \ldots, f^{k_{1}} x, x^{*}$ and hence $x^{*} \in[x]_{\tilde{G}}$. Since $f$ is orbitally G-continuous, we see that $x^{*}$ is a fixed point for $\left.f\right|_{[x]}$.

To prove (i), using (iii) we have $F(f) \neq \emptyset$ if $X^{f} \neq \emptyset$. Suppose that $F(f) \neq \emptyset$. By using the assumption that $\Delta \subseteq E(G)$, we immediately obtain $X^{f} \neq \emptyset$. Hence (i) holds.

For proving (ii) let $x \in X^{f}$. If we use weak connectivity of $G$, we have $X=[x]_{\tilde{G}}$ and by applying (iii) we obtain the desired result.

The next example illustrates that $f$ must be orbitally G-continuous in order to obtain statements which are given in Theorem 3.

Example 2 Let $X=[0,1]$ be endowed with the usual metric. Consider

$$
E(G)=\{(0,0)\} \cup\{(0, x): x \geq 1 / 2\} \cup\{(x, y): x, y \in(0,1]\},
$$


and $f: X \rightarrow X$,

$$
f x= \begin{cases}\frac{x}{2}, & \text { if } x \in(0,1] \\ \frac{1}{2}, & \text { if } x=0 .\end{cases}
$$

Then $G$ is weakly connected, $X^{f}$ is nonempty and $f$ is a $(G, \psi)$-graphic contraction where $\psi(\omega)=\frac{\omega}{3}$, but it is not orbitally $G$-continuous. Thus, $f$ does not have a fixed point.

Remark 2 In Theorem 3, by replacing the condition that the triple $(X, d, G)$ satisfies (9) and $f$ is orbitally $G$-continuous with the mapping $f$ is orbitally continuous, we have the above result, too.

The following example demonstrates that the $(G, \psi)$-graphic contraction is more general than the $(G, \psi)$-contraction.

Example 3 Let $X=[0,1]$ be endowed with the usual metric. Take

$$
E(G)=\{(0,0)\} \cup\{(0,1)\} \cup\{(x, y) \in(0,1] \times(0,1]: x \geq y\},
$$

and $f: X \rightarrow X$ as follows:

$$
f x= \begin{cases}\frac{x}{2}, & \text { if } x \in(0,1], \\ \frac{3}{4}, & \text { if } x=0 .\end{cases}
$$

Then $G$ is weakly connected and $X^{f}$ is nonempty and $f$ is a $(G, \psi)$-graphic contraction with $\psi(\omega)=\frac{\omega}{2}$ which is not a $(G, \psi)$-contraction.

Proof It is clear that $G$ is weakly connected, $X^{f} \neq \emptyset$, and with simple calculations it can be easily seen that $f$ is a $(G, \psi)$-graphic contraction. Take

$$
\psi\left(d\left(f 0, f \frac{1}{2}\right)\right) \leq c \psi\left(d\left(0, \frac{1}{2}\right)\right) \Rightarrow \frac{1}{4} \leq c \frac{1}{4},
$$

which is a contradiction since $c \in[0,1)$. Thus, $f$ is not $(G, \psi)$-contraction.

Remark 3 In Theorem 3, if we take $\psi(\omega)=\omega$, then we get Theorem 2.1, which is given in $[8]$.

\section{Competing interests}

The authors declare that they have no competing interests.

\section{Authors' contributions}

All authors contributed equally in writing this article. All authors read and approved the final manuscript.

\section{Acknowledgements}

The authors are grateful to the reviewers for their careful reviews and useful comments. 


\section{References}

1. Granas, A, Dugundji, J: Fixed Point Theory. Springer, New York (2003)

2. Jachymski, J: The contraction principle for mappings on a metric space endowed with a graph. Proc. Am. Math. Soc 136, 1359-1373 (2008)

3. Gwóźdź, LG, Jachymski, J: IFS on a metric space with a graph structure and extensions of the Kelisky-Rivlin theorem J. Math. Anal. Appl. 356, 453-463 (2009)

4. Beg, I, Butt, RA, Radojević, S: The contraction principle for set valued mappings on a metric space with a graph. Comput. Math. Appl. 60, 1214-1219(2010)

5. Bojor, F: Fixed points of Kannan mappings in metric spaces endowed with a graph. An. Univ. "Ovidius" Constanţa, Ser. Mat., 20(1), 31-40 (2012)

6. Bojor, F: Fixed point theorems for Reich type contractions on metric spaces with a graph. Nonlinear Anal. 75, 3895-3901 (2012)

7. Bojor, F: Fixed point of $\varphi$-contraction in metric spaces endowed with a graph. An. Univ. Craiova, Ser. Mat. Inform. 37(4), 85-92 (2010)

8. Petruşel, GR, Chifu, Cl: Generalized contractions in metric spaces endowed with a graph. Fixed Point Theory Appl. 2012, Article ID 161 (2012)

9. Johnsonbaugh, R: Discrete Mathematics. Prentice Hall, New Jersey (1997)

10. Shatanawi, W, Postolache, M: Common fixed point theorems for dominating and weak annihilator mappings in ordered metric spaces. Fixed Point Theory Appl. 2013, Article ID 271 (2013) doi:10.1186/1687-1812-2013-271

11. Shatanawi, W, Pitea, A: Some coupled fixed point theorems in quasi-partial metric spaces. Fixed Point Theory Appl. 2013, Article ID 153 (2013). doi:10.1186/1687-1812-2013-153

12. Shatanawi, W: Some fixed point results for a generalized $\psi$-weak contraction mappings in orbitally metric spaces. Chaos Solitons Fractals 2012(45), 520-526 (2012)

13. Shatanawi, W, Samet, B: On $(\psi, \varphi)$-weakly contractive condition in partially ordered metric spaces. Comput. Math Appl. 62, 3204-3214 (2011). doi:10.1016/j.camwa.2011.08.033

10.1186/1029-242X-2014-39

Cite this article as: Öztürk and Girgin: On some fixed-point theorems for $\psi$-contraction on metric space involving a graph. Journal of Inequalities and Applications 2014, 2014:39

\section{Submit your manuscript to a SpringerOpen ${ }^{0}$ journal and benefit from:}

- Convenient online submission

- Rigorous peer review

- Immediate publication on acceptance

- Open access: articles freely available online

- High visibility within the field

- Retaining the copyright to your article 